\title{
Morphological stability during solidification of silicon incorporating metallic impurities
}

\section{Citation}

Warrender, Jeffrey M., Jay Mathews, Daniel Recht, Matthew Smith, Silvija Gradecak, and Michael J. Aziz. 2014. "Morphological Stability During Solidification of Silicon Incorporating Metallic Impurities." Journal of Applied Physics 115 (16) (April 28): 163516. doi:10.1063/1.4871809.

\section{Published Version}

doi:10.1063/1.4871809

\section{Permanent link}

http://nrs.harvard.edu/urn-3:HUL.InstRepos:25984082

\section{Terms of Use}

This article was downloaded from Harvard University's DASH repository, and is made available under the terms and conditions applicable to Open Access Policy Articles, as set forth at http:// nrs.harvard.edu/urn-3:HUL.InstRepos:dash.current.terms-of-use\#OAP

\section{Share Your Story}

The Harvard community has made this article openly available.

Please share how this access benefits you. Submit a story.

Accessibility 
Published as Journal of Applied Physics 115, 163516 (2014).

\section{Morphological Stability During Solidification of Silicon Incorporating Metallic Impurities}

Jeffrey M. Warrender, ${ }^{1, \bullet}$ Jay Mathews, ${ }^{1, *}$ Daniel Recht, ${ }^{2}$ Matthew Smith, ${ }^{3}$ Silvija Gradečak, ${ }^{3}$ and Michael J. Aziz ${ }^{2}$

${ }^{1}$ U.S. Army ARDEC - Benét Laboratories, Watervliet, New York 12189, USA

${ }^{2}$ Harvard School of Engineering and Applied Sciences, Cambridge, Massachusetts 02138, USA

${ }^{3}$ Department of Materials Science and Engineering, Massachusetts Institute of Technology, Cambridge, Massachusetts 02139, USA

\section{ABSTRACT}

We study the stability of a planar solidification front during pulsed laser melting-induced rapid solidification of silicon containing high concentrations of ion-implanted metallic impurities. We calculate the critical impurity concentration for destabilizing plane-front solidification, and introduce the "amplification coefficient”, which is an empirical parameter describing the degree of amplification that must occur between the time the planar liquid-solid interface first becomes unstable, and the time of formation of morphological features of interface breakdown that are later observed in the microstructure. By connecting our calculations to experimental observations from the literature we determine this parameter for $\mathrm{Au}, \mathrm{Co}, \mathrm{Cr}, \mathrm{Fe}, \mathrm{Ga}$, In, and $\mathrm{Zn}$ in (100) Si and Ti in (111) Si, and find that it increases with impurity diffusive speed $v_{D}$ approximately as $v_{D}^{0.56}$. We present an approximate but simple method of estimating the maximum impurity concentration that may be incorporated in a surface layer of a given thickness without the appearance of cellular breakdown.

- Corresponding author. Electronic mail: jwarrend@post.harvard.edu

* Present address: University of Dayton, Dayton, OH. 


\section{INTRODUCTION}

The use of pulsed laser melting and rapid solidification to achieve supersaturation of conventional impurities in silicon has been extensively studied. ${ }^{1,2,3,4}$ In recent years, silicon supersaturated with sulfur has shown strong sub-band gap infrared absorption and device response. ${ }^{5,6,7}$ This has led to interest in alternative dopants, including $\mathrm{Au}, \mathrm{Zn}, \mathrm{Fe}, \mathrm{Ti}$, Co, and others. $8,9,10,11$ Although many of these are generally regarded as contaminants, most exhibit impurity states deep within the Si band gap, and if sufficiently high supersaturations can be achieved, such states would be expected to broaden into impurity bands, which could be one possible realization of intermediate band optoelectronic devices. ${ }^{12,13,14}$ Recently, sub gap device response out to $2200 \mathrm{~nm}$ was reported in a device with a Si:Au layer. ${ }^{15}$ One obstacle to fabricating Si layers rich in metal impurities is the susceptibility of the liquid/solid interface to morphological instability during solidification. The stability of an interface during alloy solidification ${ }^{16,17}$ has been treated mathematically for rapid ${ }^{18}$ solidification, and has been studied experimentally. ${ }^{19}$ The concentration gradient of the impurity in the liquid at the interface can cause the morphological instability and can give rise to the formation of a characteristic morphology known as “cellular breakdown”, so-called because of the cell-like appearance. ${ }^{20,21,22}$ In a previous work ${ }^{8}$, we reported that the depth at which breakdown was achieved was closer to the surface than the (calculated) depth at which the critical concentration for instability was exceeded, suggesting a delay between the time that a perturbation forms and the time at which it has amplified enough to exhibit morphological features that are detectable by examination of the microstructure or composition profile. We conclude that the amplification rate of the initial perturbation plays an important role in the phenomenology of breakdown. 
Practical realization of layers containing high supersaturations of transition metal impurities requires knowledge about the maximum concentration that can be incorporated, and the maximum layer thickness that can be achieved without breakdown. In this work, we calculate the stability of the interface during rapid solidification for $\mathrm{Au}, \mathrm{In}, \mathrm{Ga}, \mathrm{Co}, \mathrm{Cr}, \mathrm{Fe}$, and $\mathrm{Zn}$ in (100) Si and Ti in (111) Si. We show that an unstable concentration in the liquid does not immediately result in breakdown of the interface, but that, by calculating the characteristic time for a perturbation to amplify, we can describe the onset of cellular breakdown in terms of an “amplification parameter”. We also provide a simple method to approximate the maximum thickness that can be achieved given a desired impurity concentration, and vice versa.

Mullins and Sekerka ${ }^{16}$ showed that, for a perturbation of spatial frequency $\omega$ and time-dependent amplitude $\delta(t)$ at the planar interface during solidification of a liquid, there are three contributions to the growth or suppression of the perturbation. If most of the latent heat of solidification is being removed through the solid, as it is during pulsed laser melting (PLM), the thermal gradient in the liquid or solid is always stabilizing, and is the primary stabilizing contribution at very low velocity of the liquid/solid interface. Capillarity also will always act to suppress the perturbation, and is the primary stabilizing contribution at very high interface velocity.

Working against these to destabilize the liquid-solid interface is the concentration gradient, which forms in the liquid just ahead of the interface due to partitioning of the impurity at the interface. For all impurities of interest in this work, the equilibrium concentration of impurity in the solid is lower than in the liquid. Therefore, the impurity tends to be rejected by the solid, but requires time to diffuse into the bulk of the liquid, which creates a concentration gradient in the liquid, with the highest concentration in the liquid at the interface. The higher the concentration, 
the higher the impurity-induced depression of the melting point. Thus, when a bit of the solidification front jumps ahead of neighboring regions in a fluctuation, the peak advances into a liquid of higher melting point, finds itself undercooled, and accelerates. The sweeping of impurity normal to the interface along the sides of the advancing peak act to depress the melting point of the troughs in between the peaks and thereby slow their solidification. The slowing of the troughs reduces the kinetic partition coefficient, enhancing the effect. Eventually, the concentration of impurity may become so high that precipitates or liquid droplets form. ${ }^{3}$ Because growth of the solid proceeds normally to the interface, the troughs eventually slow down so much that growth on either side of the troughs is perpendicular to the original solidification direction, and when two perpendicular solidification fronts from either side of a trough join, the characteristic cellular morphology is created.

The concentration gradient therefore acts to amplify the perturbation, and the relative magnitude of this contribution vis à vis the stabilizing influences of capillarity and the thermal gradient determines whether the perturbation of spatial frequency $\omega$ is amplified or decays. Mullins and Sekerka obtained a growth rate for $\delta$,

$$
\frac{\dot{\delta}}{\delta}=\frac{v \omega\left\{\left[-2 T_{M} \Gamma \omega^{2}-\left(\mathcal{G}^{\prime}+\mathcal{G}\right)\right]\left[\omega^{*}-\left(V / D_{l i q}\right) p\right]+2 m G_{c}\left[\omega^{*}-\left(V / D_{l i q}\right)\right]\right\}}{\left(\mathcal{G}^{\prime}-\mathcal{G}\right)\left[\omega^{*}-\left(V / D_{l i q}\right) p\right]+2 \omega m G_{C}},
$$

with $k$ the partition coefficient, $p=1-k, T_{M}$ the liquid melting temperature, $v$ the interface speed, $D_{\text {liq }}$ the diffusivity of the impurity in liquid $\mathrm{Si}$, $\Gamma$ the interfacial tension divided by $L_{M}$ the latent heat of fusion, $m$ the liquidus slope, $\mathcal{G}\left(\mathcal{G}^{\prime}\right)$ the normalized thermal gradient in the liquid (solid),

$$
\omega^{*} \equiv\left(\frac{V}{2 D_{l i q}}\right)+\left[\left(\frac{V}{2 D_{l i q}}\right)^{2}+\omega^{2}\right]^{1 / 2}
$$


and

$$
m G_{c}=\frac{v(k-1)}{D_{l i q} k} m c_{\infty}
$$

with $c_{\infty}$ the bulk liquid concentration. Following Mullins and Sekerka, we set $\mathcal{G}^{\prime}=\mathcal{G}+$ $v L_{M} / \kappa_{a v g}$, with $\kappa_{a v g}$ the average of the liquid and solid thermal conductivities. We refer to $\dot{\delta} / \delta$ as the "amplification rate", and its inverse, $\tau$, as the "amplification time", the time required for the perturbation amplitude to grow by a factor of $e$.

Although these equations were derived for low solidification velocity, with appropriate corrections their validity can be extended to rapid solidification as well. Aziz and Kaplan demonstrated that, at high solidification velocities, the partition coefficient $k$ is increased, according to (for a dilute solution) ${ }^{23,24}$

$$
k=\frac{k_{e q}+\frac{v}{v_{D}}}{1+\frac{v}{v_{D}}},
$$

where $v_{D}$ is the "diffusive speed", the ratio of the interface diffusivity of the impurity to the width of the interface, and $k_{e q}$ the equilibrium partition coefficient. The diffusive speed has been discussed extensively in a recent paper by Recht et al. ${ }^{8}$ Related to this, the kinetic liquidus slope becomes

$$
m(v)=m_{e q}\left\{1+\frac{k_{e q}-k(v)\left\{1-\ln \left[\frac{k(v)}{k_{e q}}\right]\right.}{1-k_{e q}}\right\}
$$

with $m_{e q}$ the equilibrium liquidus slope, which we obtain using 
$m_{e q}=(1-k) R T_{M}{ }^{2} / L_{M} \cdot{ }^{19}$ Together, these constitute the "Continuous Growth Model (CGM)" for partitioning during rapid solidification. ${ }^{24,4}$

Sekerka showed how the numerator of the right hand side of Eq. (1) can be recast as a cubic equation, whose real, positive root can be manipulated algebraically to obtain $c_{\infty}^{*}$, the critical bulk liquid concentration. ${ }^{17}$ Fig. 1 illustrates the relationship between $c_{\infty}$, the perturbation spatial frequency $\omega$, and the amplification rate $\dot{\delta} / \delta$. When $c_{\infty}$ equals $c_{\infty}^{*}$, the amplification rate is zero at the most unstable spatial frequency (the local maximum of the amplification rate curve). As $c_{\infty}$ increases beyond $c_{\infty}^{*}$, the most unstable spatial frequency increases, and the amplification rate becomes positive over a range of frequencies, with the amplification rate at the most unstable frequency increasing with increasing $c_{\infty}$. Correspondingly, this implies that the amplification time $\tau$ decreases with increasing $c_{\infty}$.

We seek to compare the results of such calculations with experimental observations of cellular breakdown for Si containing $\mathrm{Sn}^{19}, \mathrm{Ga}^{21}$, $\mathrm{Au}, \mathrm{Fe}, \mathrm{Co}, \mathrm{Cr}, \mathrm{Zn}^{8}, \mathrm{In}^{25}$, and $\mathrm{Ti}^{9}$.

\section{DATA AND ANALYSIS}

\section{A. Stability Calculation}

We first used the aforementioned method of Mullins and Sekerka, with the CGM corrections, to obtain the predicted critical bulk liquid concentration $c_{\infty}^{*}$ (expressed as an atomic fraction), as a function of interface velocity for all impurities, using thermal parameters provided in Table I and impurity-specific parameters provided in Table II. The results are presented in the left column of

Fig. 2. Additionally, using equation (1), and the definition of $\tau$ as the inverse of $\dot{\delta} / \delta$, we calculated the amplification time $\tau$ as a function of the ratio $c_{\infty} / c_{\infty}^{*}$ for two representative interface velocities, $v=1 \mathrm{~m} / \mathrm{s}$ and $v=10 \mathrm{~m} / \mathrm{s}$, and the resulting values are presented in the center 6 
and right columns of Fig. 2, respectively. The diffusive speed $v_{D}$ is generally obtained by performing a $1 \mathrm{D}$ diffusion calculation ${ }^{26}$ and adjusting $v_{D}$ until good agreement between the predicted impurity depth profile and an experimental depth profile obtained from Secondary Ion Mass Spectrometry (SIMS) or Rutherford Backscattering Spectrometry (RBS) is achieved. Uncertainty arising from the calibration of the depth and concentration axes in the depth profiles creates uncertainty in the calculated $v_{D}$, and a possible error of up to a factor of 2 can be assumed. Therefore, for each impurity, we calculated $c_{\infty}^{*}$ and $\tau$ for the literature value of $v_{D},{ }^{25}$ and also for a value a factor of 2 higher and lower. Results for all three values are presented for each impurity.

The left column presents the neutral stability condition for each impurity; growth under conditions (interface speed and bulk liquid concentration) that fall below the curve are stable, and above the curve, unstable. The plots in this column therefore identify the conditions under which stable growth can be expected. The center and right columns are calculated for the most unstable spatial frequency $\omega$ corresponding to the interface speed $v$ for that column.

Although the middle and right columns of Fig. 2 show that amplification times are shorter for $v=10 \mathrm{~m} / \mathrm{s}$ compared to $1 \mathrm{~m} / \mathrm{s}$, it must be remembered that the abscissa of these columns is normalized bulk liquid concentration, and the stable bulk liquid concentration will be greater at higher speed; thus, these columns show the effect of excess concentration on the amplification time.

\section{B. Cellular Breakdown}

Next, we seek to connect these calculations with the experimentally observed onset of cellular breakdown in layers containing these impurities. Breakdown manifests in two ways - visually, 
the cell walls are observable in plan view using the backscatter detector in a scanning electron microscope (SEM) or in cross section using a Transmission Electron Microscope (TEM); and, compositionally, the onset of breakdown is indicated by an abrupt increase in impurity concentration with decreasing depth in the solid, resulting from excess impurity being trapped in cell walls. Hoglund noted that the latter effect can be detected deeper in the layer can than the former effect. ${ }^{19}$ We use literature results, which were obtained using microscopy for Ga and composition for all other impurities, to set an experimental "breakdown depth" $d_{B}$ for each impurity. The ion implantation and laser melting parameters that were used to fabricate these samples are provided in Table III, as are the literature values of $d_{B}$.

Using experimental depth profiles (when available) or SRIM calculations (when not) for the initial concentration depth profile, ${ }^{27}$ and the results of experimentally validated $1 \mathrm{D}$ heat flow calculations ${ }^{19}$ to estimate the thermal conditions that each sample experienced, we use a numerical solution of the $1 \mathrm{D}$ diffusion equation with partitioning at a moving boundary ${ }^{26}$ to predict the concentration depth profile that each sample would have experienced under the conditions given in Table III. We seek the time required for amplification of a perturbation that forms when the interface is at any given depth $d$. To make this calculation tractable, we make several simplifying assumptions. (i) The spatial frequency of the perturbation that forms at $d$ is unique, and is the most unstable frequency at that depth. (ii) The growth of this perturbation, having spatial frequency $\omega(d)$, is unaffected by the growth of other perturbations and their associated spatial frequencies. (iii) The perturbation grows at a constant rate, given by $\dot{\delta} / \delta$, which, according to equation (1), is a function of $\omega(d)$, of $v(d)$, the interface speed at $d$, and of the ratio $c_{\infty} / c_{\infty}^{*}$ at $d$. The growth can therefore be described by a characteristic time for amplification by a factor of $e, \tau$, which is the inverse of $\dot{\delta} / \delta$. (iv) By the time the perturbation 
has amplified by a factor of $e$, the interface will have traveled toward the surface by a distance $\ell=v(d) \tau$. (v) To obtain $c_{\infty} / c_{\infty}^{*}, c_{\infty}$ can be approximated by a time-independent curve, and (vi) $c_{\infty}(d)$ can be obtained from that curve by adding a constant, impurity-specific (negative) offset to $d$, which approximately corresponds to the spatial extent of the tail of the interface concentration gradient extending into the liquid (essentially, it is the "distance" from the interface to the bulk liquid). With these assumptions, we obtain a unique value of $\tau$ for each $d$. Fig. 3(a) illustrates the method, using representative data from a calculation of In in Si under the conditions given in Table II.

Assumptions (v) and (vi) are made for computational convenience, but are justified by examination of Fig. 3(c), which shows the concentration depth profile of In in Si for several different time steps during solidification. The liquid concentration beyond the tail of the concentration peak is approximately uniform for all time steps, and is slowly-varying; therefore, assuming a constant offset value, while technically inaccurate, ${ }^{28}$ only contributes a small possible error, which we estimate below. Assumption (iii), and its derivative assumption, (iv), are not realized in the actual solidification, because the amplification rate at a fixed frequency increases as the ratio $c_{\infty} / c_{\infty}^{*}$ increases; and additionally, the speed changes with depth. However, if we allow assumption (i) to hold, we find that the amplification rate quickly converges to a maximum of a factor of between 1.4-2.2 of its initial value (impurity-dependent) as $c_{\infty} / c_{\infty}^{*}$ is increased, assuming constant speed; if the decreasing speed is taken into account in the calculated amplification rate, this factor is even smaller. And, the speed decreases by less than $20 \%$ between the depth at which $c_{\infty}^{*}$ is achieved in the liquid and the depth at which a perturbation is observed. Therefore, treating $v$ and $\tau$ as constant are reasonable approximations that have the advantage of making the subsequent calculations tractable. Finally, we acknowledge that in the 
actual solidification, all wavelengths are present simultaneously, and that assumption (ii) is only strictly true when the amplitudes remain small.

\section{Amplification Coefficient}

Using the approach described above, we can calculate a curve for $\tau$ as a function of depth, and can use it to calculate a predicted breakdown depth vs. $d$. Here we face the complication that we do not know a priori how many periods of amplification will be required for breakdown to be achieved. We introduce a quantity $\xi$ as an empirical parameter, which we refer to as the "amplification coefficient". For a perturbation that first amplifies when the interface is at depth $d$, the depth at which breakdown would be expected occur is $d-v \xi \tau$, where $v$ and $\tau$ depend on $d$. We plot the results of this calculation in Fig. 4 for the case of In. The solid line corresponds to $c_{\infty}$, and sets the value of $d$ for the plot of the expected breakdown depth curve. The abscissa of the breakdown depth curve is $d-v \xi \tau$, and the ordinate is the bulk liquid concentration $c_{\infty}$ at the time that the interface was at depth $d$. In other words, the horizontal distance between the breakdown depth curve and the bulk liquid concentration curve, for a given concentration, is the "breakdown length”, $\nu \xi \tau$, which is the distance the interface will have traveled before sufficient amplification has occurred for breakdown. The predicted breakdown depth, then, will be the right-most (i.e., deepest) point on the breakdown depth curve, and this can be compared to the observed thickness of the broken-down layer.

Fig. 4 has several features of note. First, as mentioned in a previous paper $^{8}$, the experimental breakdown depth does not occur at the crossing of $c_{\infty}$ and $c_{\infty}^{*}$, validating the intuition that the perturbation would need to amplify before breakdown is observable. Second, because we assumed a constant value for $v$ and $\tau$, we can vary $\xi$ until we achieve agreement between the 
breakdown curve and the experimental breakdown depth. For In diffusion simulated using the conditions of Table II, we find that $\xi=7.8$ gives agreement with the experimental breakdown depth $d_{B}$ of $106 \mathrm{~nm}$.

Before we can fully generalize this method for all impurities, we first consider a single impurity at several implantation doses, to ascertain whether $\xi$ has a dose dependence. In Ref. 9, we reported on the behavior of $\mathrm{Ti}$ in $\mathrm{Si}$ (111) at various implantation doses. Identical substrate, implantation energy, and laser melting conditions were used for all doses, making this system ideal for this comparison. In this work, we considered four implantation doses: $1 \times 10^{16}$ at./cm², $3 \times 10^{15}$ at. $/ \mathrm{cm}^{2}, 8 \times 10^{14}$ at. $/ \mathrm{cm}^{2}$, and $1 \times 10^{14}$ at. $/ \mathrm{cm}^{2}$. As reported in Table III, all except $1 \times 10^{14}$ at./ $\mathrm{cm}^{2}$ showed a broadened surface concentration peak in SIMS depth profiles, which is known to be characteristic of cellular breakdown. ${ }^{19,21}$ We used SIMS as-implanted profiles for the initial depth profile, and generated a melt depth vs. time curve using the intensity profile of the Nd:YAG laser. Using these, we generated a $c_{\infty}^{*}$ and $c_{\infty}$ curve for each dose, using the best-fit value of $v_{D}$ obtained from fitting the post-melting SIMS profile for that dose. Finally, setting $\xi$ to 4.06, we calculated the breakdown depth curve $d-v \xi \tau$ for each dose, using $c_{\infty}(d)$ for that dose as the ordinate. Fig. 5 shows the resulting curves, along with vertical lines corresponding to the breakdown depths observed in SIMS data. The agreement between the rightmost point of each breakdown depth curve and the observed experimental breakdown depth for that same dose is excellent. (Note particularly that the lowest dose, $1 \times 10^{14} \mathrm{at} . / \mathrm{cm}^{2}$, did not show breakdown experimentally, and in Fig. 5, its breakdown depth curve has a negative abscissa for all concentrations, indicating that, for all $c_{\infty}(d), v \xi \tau>d$, and therefore, the layer would not have been expected to show breakdown.) We conclude that there is no value to be added to the model by including a dose-dependence to $\xi$. 
We followed a similar method to obtain the amplification coefficient for all of the impurities previously mentioned, and present the results in Table IV. These may be used in the simple method described in the next section to estimate the critical thickness for breakdown for these impurities.

We identify two potential sources of error in $\xi$. First, we estimate the error in the offset from $d$ as at most $20 \%$, which results in an error in $\xi$ of about $6 \%$. Second, using Fig. 2, at $1 \mathrm{~m} / \mathrm{s}$, an error in $v_{D}$ of a factor of 2 could result in an error in $\tau$ on the order of $20 \%$ (for Au), which could result in an adjustment to $\xi$ of $16 \%$. Adding these two estimates together in quadrature, we estimate a maximum total error of $17 \%$ in our calculated values of $\xi$.

We can corroborate our observation by comparing the most unstable wavelength $(\lambda=2 \pi / \omega)$ at $d=d_{B}+v \xi \tau$, the depth at which the "breakdown perturbation" formed, to the average cell wall spacing observed in post mortem microscopy of the broken-down layer. Narayan reports experimental cell wall spacings of $50 \mathrm{~nm}$ and $70 \mathrm{~nm}$ for In and Ga, respectively, under comparable conditions to those of our calculations. ${ }^{21}$ For In, under our model, the breakdown perturbation first amplified when the interface was at a depth of $180 \mathrm{~nm}$, and $\lambda=28 \mathrm{~nm}$; for Ga, the corresponding values are $120 \mathrm{~nm}$ and $57 \mathrm{~nm}$, respectively. These results are in reasonably good agreement with the experimental observations. Exact agreement of the periodicity would have resulted had the breakdown perturbations first amplified at $230 \mathrm{~nm}$ (In) and $150 \mathrm{~nm}$ (Ga), in the model.

D. Predicting the Critical Thickness

A practical question confronting the experimentalist or the technologist pertains to the maximum thickness that can be achieved for a given concentration of a particular impurity before the 
observation of breakdown, or the maximum concentration that can be achieved for a given thickness. We now provide a simple method for estimating this. We first acknowledge that direct experimental observation of the bulk liquid concentration is impractical. We use 1D diffusion simulations to estimate a quantity $\zeta$, with $\zeta \equiv c_{\infty}^{\max } / c_{s}^{\max }$, with $c_{s}^{\max }$ the maximum solid concentration after solidification is complete, disregarding the concentration peak near the surface that results from the "snowplowing" of impurity to the surface during partitioning. Fig. 7 shows, graphically, our method for obtaining $c_{\infty}^{\max }$ and $c_{s}^{\max }$. We present the values of $\zeta$ for each impurity in Table IV. Unlike $\xi$, $\zeta$ may have a weak dose dependence; our values for Ti changed by slightly less than a factor of 2 between the $1 \times 10^{16}$ at./ $\mathrm{cm}^{2}$ dose and the $8 \times 10^{14}$ at. $/ \mathrm{cm}^{2}$ dose. But as a tool for approximating, we believe $\zeta$ is nevertheless useful.

Assuming one has a target concentration $c_{S}$ and the ability to achieve a (fixed) interface velocity $v$, the following method is used to obtain the critical thickness of a layer that does not exhibit breakdown. First, Fig. 2 is used to obtain the critical bulk liquid concentration $c_{\infty}^{*}$. Next, $c_{S}$ is multiplied by $\zeta$ (from Table IV) to provide a target bulk liquid concentration $c_{\infty}$. Assuming for simplicity that $v$ is either $1 \mathrm{~m} / \mathrm{s}$ (excimer) or $10 \mathrm{~m} / \mathrm{s}$ (Nd:YAG), the appropriate column of Fig. 2 is then used to obtain $\tau$ at $c_{\infty} / c_{\infty}^{*}$. Using $\xi$ from Table IV, the critical thickness is simply

$$
d_{c r i t}=v \xi \tau
$$

To obtain the maximum achievable concentration for a given layer thickness, the reverse process is used.

As a simple example, we consider the case of a layer implanted with Au and irradiated with an Nd:YAG laser, which provides an interface velocity of $6 \mathrm{~m} / \mathrm{s}$. As an approximation, we round this up to $10 \mathrm{~m} / \mathrm{s}$ so as to be able to use the data in Fig. 2. The critical bulk liquid concentration 
$c_{\infty}^{*}$ is $3.6 \times 10^{-4}$ (atomic fraction). Supposing we seek a layer concentration of $5 \times 10^{20}$ at. $/ \mathrm{cm}^{3}$, we use the atomic density of solid $\mathrm{Si}\left(5 \times 10^{22}\right.$ at. $\left./ \mathrm{cm}^{3}\right)$ and the value of $\zeta$ for Au, 12, to calculate $c_{\infty}=0.12$, and $c_{\infty} / c_{\infty}^{*}=333$; using the $10 \mathrm{~m} / \mathrm{s}$ graph in Fig. 2, we obtain $\tau=0.063 \mathrm{~ns}$. Thus, our maximum layer thickness, using equation (6), is $d_{c r i t}=9.1 \mathrm{~nm}$. (Had we performed the calculation using data for $6 \mathrm{~m} / \mathrm{s}$, the result would have been $8.2 \mathrm{~nm}$ ). If we switch substrates, to Si (111), for which $v_{D}<200 \mathrm{~m} / \mathrm{s}$, then $\tau$ becomes $0.09 \mathrm{~ns}$, and $d_{\text {crit }}=13 \mathrm{~nm}$.

For an approximate experimental validation, we consider the case of Fig. 2 in Ref. 8, for a Si:Au layer irradiated with an excimer laser $(v=3 \mathrm{~m} / \mathrm{s})$, for which the critical bulk liquid concentration $c_{\infty}^{*}$ is $2.0 \times 10^{-5}$ (atomic fraction). Assuming a desired concentration of $2 \times 10^{19}$ at. $/ \mathrm{cm}^{3}, c_{\infty} /$ $c_{\infty}^{*}=240$, and $\tau$ is approximately $2.0 \mathrm{~ns}$, giving an estimated maximum layer thickness $d_{\text {crit }}=86$ $\mathrm{nm}$. The sample in Ref. 8 exhibits a solid concentration of $2 \times 10^{19}$ at. $/ \mathrm{cm}^{3}$ at a depth of about $220 \mathrm{~nm}$, and the depth at which breakdown is observed, $d_{B}$, is about $150 \mathrm{~nm}$, for a distance of 70 $\mathrm{nm}$, in reasonable agreement with our approximate calculation.

\section{E. Relationship between $\xi$ and $v_{D}$}

Finally, we ask whether any systematic dependence of $\xi$ across impurities is observed. In Fig. 7, we plot $\xi$ as a function of $v_{D}$ for those impurity layers fabricated in (100) material. (We exclude Ti from this plot because its calculated $v_{D}$ would likely be different were it implanted into (100) material ${ }^{26}$, and $c_{\infty}^{*}, \tau$, and $\xi$ would therefore be different as well). Per section II.C above, error bars corresponding to $17 \%$ of $\xi$, along with horizontal error bars of a factor of $+/-2$, are included in Fig. 7. 
If the Sn data point from Ref. 19 is excluded, we observe a strong correlation between $\xi$ and $v_{D}$ for those layers that were grown in (100) Si. A fit with a power law dependence $\xi \sim v_{D}^{0.56}$ gives an $\mathrm{R}^{2}$ value of 0.96 . A weak correlation between $\xi$ and $D_{\text {liq }}$ or $k_{e q}$ may be present as well. We are uncertain why the Sn data point from Ref. 19 does not fall on the curve, but we note that we were unable to reproduce their empirical melt depth of $320 \mathrm{~nm}$ in 1D heat flow calculations using all of the sample and laser parameters that they provided; we instead calculate a melt depth of $180 \mathrm{~nm}$. A deeper melt would result in a slower interface, which would lower $c_{\infty}^{*}$ and, correspondingly, would lower $\tau$, therefore, a higher value of $\xi$ would have resulted.

\section{SUMMARY}

In summary, we calculated the critical bulk liquid concentration for interface stability during solidification of laser-melted (100) silicon containing Au, Co, Cr, Fe, Ga, In, or Zn, and for (111) silicon containing Ti. We found that the interface does not break down immediately upon achievement of a bulk liquid concentration in excess of the critical concentration, but that instead, a perturbation that forms at some depth $d$ will require an impurity-dependent length for amplification, given by $v \xi \xi$, where $v$ and $\tau$ are the interface speed and inverse amplification rate at depth $d$, respectively, and $\xi$ is the empirical "amplification coefficient". We found that $\xi$ has a statistically significant power law dependence on the diffusive speed $v_{D}$, with a power of 0.56 . We also observed a tentative correlation between $\xi$ and the ratio of the maximum bulk liquid concentration to the maximum solid concentration after solidification. More substantial theoretical work will be required in order to explain the dependence of $\xi$ on $v_{D}$. A simple method for estimating the maximum concentration or layer thickness that can be achieved for a given impurity and interface speed was provided. 


\section{REFERENCES}

1. C.W. White, S.R. Wilson, B.R. Appleton, and F.W. Young, Journal of Applied Physics 51, 738 (1980).

2. M. Finetti, P. Negrini, S. Solmi, and D. Nobili, Journal of the Electrochemical Society 128, 1313 (1981).

3. C.W. White, Journal de Physique 44, C5-145 (1983).

4. J.A. Kittl, P.G. Sanders, M.J. Aziz, D.P. Brunco, and M.O. Thompson, Acta materialia 48, 4797 (2000).

5. T.G. Kim, J.M. Warrender, and M.J. Aziz, Applied Physics Letters 88, 241902 (2006).

6. B.P. Bob, A. Kohno, S. Charnvanichborikarn, J.M. Warrender, I. Umezu, M. Tabbal, J.S. Williams, and M.J. Aziz, Journal of Applied Physics 107, 123506 (2010).

7. A.J. Said, D. Recht, J.T. Sullivan, J.M. Warrender, T. Buonassisi, P.D. Persans, and M.J. Aziz, Applied Physics Letters 99, 073503 (2011).

8. D. Recht, M.J. Smith, S. Charnvanichborikarn, J.T. Sullivan, M.T. Winkler, J. Mathews, J.M. Warrender, T. Buonassisi, J.S. Williams, S. Gradečak, and M.J. Aziz, Journal of Applied Physics 114, 124903 (2013).

9. J. Mathews, A.J. Akey, D. Recht, G. Malladi, H. Efstathiadis, M.J. Aziz, and J.M. Warrender, Applied Physics Letters, in press.

10. J. Olea, M. Toledano-Luque, D. Pastor, E. San-Andrés, I. Mártil, and G. González-Díaz, Journal of Applied Physics 107, 103524 (2010).

11. Y. Zhou, F. Liu, and X. Song, Journal of Applied Physics 113, 103702 (2013).

12. A. Luque and A. Martí, Physical Review Letters 78, 5014 (1997). 
13. J.J. Krich, B.I. Halperin, and A. Aspuru-Guzik, Journal of Applied Physics 112, 013707 (2012).

14. J.T. Sullivan, C.B. Simmons, J.J. Krich, A.J. Akey, D. Recht, M.J. Aziz and T. Buonassisi, Journal of Applied Physics 114, 103701 (2013).

15. J.P. Mailoa, A.J. Akey, C.B. Simmons, D. Hutchinson, J. Mathews, J.T. Sullivan, D. Recht, M.T. Winkler, J.S. Williams, J.M. Warrender, P.D. Persans, M.J. Aziz, and T. Buonassisi, Nature Communications 5, 3011 (2014)

16. W.W. Mullins and R.F. Sekerka, Journal of Applied Physics 35, 444 (1964)

17. R.F. Sekerka, Journal of Applied Physics 36, 264 (1965).

18. J.W. Cahn, S.R. Coriell, and W.J. Boettinger, in Laser and Electron Beam Processing of Materials, edited by C.W. White and P.S. Peercy, (Academic Press, New York, 1980), p.89.

19. D.E. Hoglund, M.O. Thompson, and M.J. Aziz, Physical Review B 58, 189 (1998).

20. A.G. Cullis, D.T.J. Hurle, H.C. Webber, N.G. Chew, J.M Poate, P. Baeri, and G. Foti, Applied Physics Letters 38, 642 (1981).

21. J. Narayan, Journal of Applied Physics 52, 1289 (1981).

22. J. Narayan, Journal of Crystal Growth 59, 583 (1982).

23. We performed simulations in which the non-dilute form of $k$ was used, but found little difference between the retained impurity concentrations for the two cases.

24. M.J. Aziz and T. Kaplan, Acta metallurgica 36, 2335 (1988).

25. Data and best fit $v_{D}$ for In from D. Recht and M.J. Aziz, unpublished.

26. R. Reitano, P.M. Smith, and M.J. Aziz, Journal of Applied Physics 76, 1518 (1994).

27. www.srim.org

28. The decay length of the spike in reality equals $D_{l i q} / v$ in the quasistationary approximation. 


\begin{tabular}{|l|l|l|}
\hline \multicolumn{1}{|c|}{$\mathcal{G}$} & Thermal gradient in liquid & $2 \times 10^{4} \mathrm{~K} / \mathrm{m}$ \\
\hline$T_{M}$ & Melting temperature of Si & $1685 \mathrm{~K}$ \\
\hline$L_{M}$ & $\begin{array}{l}\text { Latent heat of fusion at melting } \\
\text { temperature }\end{array}$ & $4.19 \times 10^{9} \mathrm{~J} / \mathrm{m}^{3}$ \\
\hline$\gamma\left(=\Gamma L_{M}\right)$ & Interfacial tension & $0.36 \mathrm{~J} / \mathrm{m}^{2}$ \\
\hline
\end{tabular}

Table I. Parameters used in calculations of $c_{\infty}^{\max }$. All values from Ref. 19. 


\begin{tabular}{|l|l|l|l|l|}
\hline Impurity & $v_{\mathrm{D}}(\mathrm{m} / \mathrm{s})$ & $D_{\text {liq }}\left(\mathrm{cm}^{2} / \mathrm{s}\right)$ & $k_{e q}$ & Offset $(\mathrm{nm})$ \\
\hline $\mathrm{Au}^{8}(*)$ & 350 & $6 \times 10^{-5}$ & 0.000025 & 30 \\
\hline $\mathrm{Co}^{8}$ & 10000 & $1.8 \times 10^{-5}$ & 0.00001 & 5 \\
\hline $\mathrm{Cr}^{8}$ & 10000 & 1 & 0.000011 & 5 \\
\hline $\mathrm{Fe}^{8}$ & 100 & $3.2 \times 10^{-5}$ & 0.000007 & 10 \\
\hline $\mathrm{Ga}^{21}$ & 35 & $3.6 \times 10^{-4}$ & 0.008 & 30 \\
\hline $\mathrm{In}^{26}(*)$ & 100 & $1.6 \times 10^{-4}$ & 0.0004 & 25 \\
\hline $\mathrm{Sn}^{19}$ & 17 & $2.5 \times 10^{-4}$ & 0.016 & \\
\hline $\mathrm{Ti}^{9}$ & 20000 & $6 \times 10^{-4}$ & 0.000002 & 20 \\
\hline $\mathrm{Zn}^{8}$ & 1000 & $3.2 \times 10^{-4}$ & 0.00001 & 30 \\
\hline
\end{tabular}

Table II. Parameters used in calculation of thermal properties and in diffusion calculations. $\left(^{*}\right)$ For $\mathrm{Au}$ and In, the literature values reported in Table II were used to generate Fig. 2 (note that the literature value for $v_{D}$ for In is $85.5 \mathrm{~m} / \mathrm{s}^{26}$ ); for all other calculations, the best-fit values from 1D diffusion simulations in comparison with SIMS measurements were used, namely, $D_{\text {liq }}=$ $3 \times 10^{-4} \mathrm{~cm}^{2} / \mathrm{s}$ (both), and $v_{D}=400 \mathrm{~m} / \mathrm{s}(\mathrm{Au})$ and $200 \mathrm{~m} / \mathrm{s}$ (In). 


\begin{tabular}{|l|l|l|l|l|l|l|}
\hline Impurity & Orientation & $\begin{array}{l}\text { Ion energy } \\
(\mathrm{keV})\end{array}$ & $\begin{array}{l}\text { Implantation } \\
\text { dose }\left(\mathrm{at}^{\prime} \mathrm{cm}^{2}\right)\end{array}$ & Laser & $\begin{array}{l}\text { Laser } \\
\text { fluence } \\
\left(\mathrm{J} / \mathrm{cm}^{2}\right)\end{array}$ & $d_{B}(\mathrm{~nm})$ \\
\hline $\mathrm{Au}^{8}$ & $(100)$ & 325 & $1 \times 10^{16}$ & $\mathrm{XeCl}$ & 2.0 & 150 \\
\hline $\mathrm{Co}^{8}$ & $(100)$ & 120 & $1 \times 10^{16}$ & $\mathrm{XeCl}$ & 2.0 & 150 \\
\hline $\mathrm{Cr}^{8}$ & $(100)$ & 95 & $5 \times 10^{15}$ & $\mathrm{XeCl}$ & 2.0 & 110 \\
\hline $\mathrm{Fe}^{8}$ & $(100)$ & 140 & $1 \times 10^{16}$ & XeCl & 2.0 & 140 \\
\hline $\mathrm{Ga}^{21}$ & $(100)$ & 100 & $1.2 \times 10^{16}$ & Ruby $\left(^{*}\right)$ & 1.5 & 50 \\
\hline $\mathrm{In}^{25}$ & $(100)$ & 160 & $1 \times 10^{16}$ & XeCl & 2.0 & 106 \\
\hline $\mathrm{Sn}^{19}$ & $(100)$ & 120 & $3.5 \times 10^{15}$ & XeCl & 1.25 & 70 \\
\hline $\mathrm{Ti}^{9}$ & $(111)$ & 15 & $\begin{array}{l}1 \times 10^{16} \\
3 \times 10^{15}, \\
8 \times 10^{14}, \\
1 \times 10^{14}\end{array}$ & Nd:YAG & 0.75 & 70,50, \\
& & & $5 \times 10^{15}$ & & & $25, \mathrm{~N} / \mathrm{A}$ \\
\hline $\mathrm{Zn}^{8}$ & $(100)$ & 120 & & & 2.0 & 43 \\
\hline
\end{tabular}

Table III. Experimental parameters for impurity implantation, laser melting, and observed breakdown depth $d_{B}$. For lasers, pulse intensity profiles were obtained from the actual lasers used, with the exception of (*) Ruby, for which a Gaussian pulse with FWHM 15 ns was used. 


\begin{tabular}{|l|l|l|}
\hline Impurity & $\xi$ & $\zeta$ \\
\hline $\mathrm{Au}$ & 14.4 & 12 \\
\hline $\mathrm{Co}$ & 82.6 & 23 \\
\hline $\mathrm{Cr}$ & 107.9 & 16 \\
\hline $\mathrm{Fe}$ & 7.4 & 1.3 \\
\hline $\mathrm{Ga}$ & 3.6 & 1.6 \\
\hline $\mathrm{In}$ & 7.8 & 5.6 \\
\hline $\mathrm{Ti}$ & 4.06 & $450\left(1 \times 10^{16} \mathrm{at} . / \mathrm{cm}^{2}\right)$ \\
& & $660\left(3 \times 10^{15} \mathrm{at} / \mathrm{cm}^{2}\right)$ \\
& & $20\left(8 \times 10^{14} \mathrm{at} . / \mathrm{cm}^{2}\right)$ \\
\hline $\mathrm{Zn}$ & 28.8 & 20 \\
\hline
\end{tabular}

Table IV. Best values of $\xi$ and $\zeta$ for each impurity. 


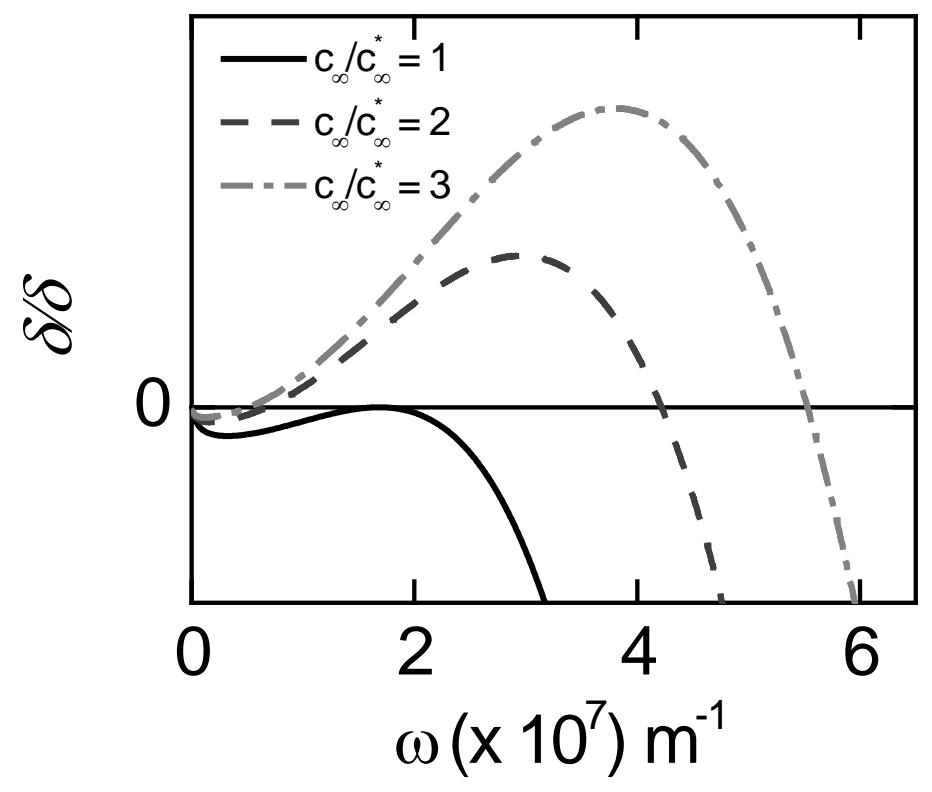

Fig. 1. Amplification rate vs. perturbation spatial frequency $\omega$ at bulk liquid concentrations 1 (solid), 2 (dash), and 3 (dash-dot) times the critical bulk liquid concentration. As the bulk liquid concentration exceeds the critical concentration, the amplification rate becomes positive, and a perturbation will grow. The most unstable wavelength becomes shorter with increasing excess concentration. 


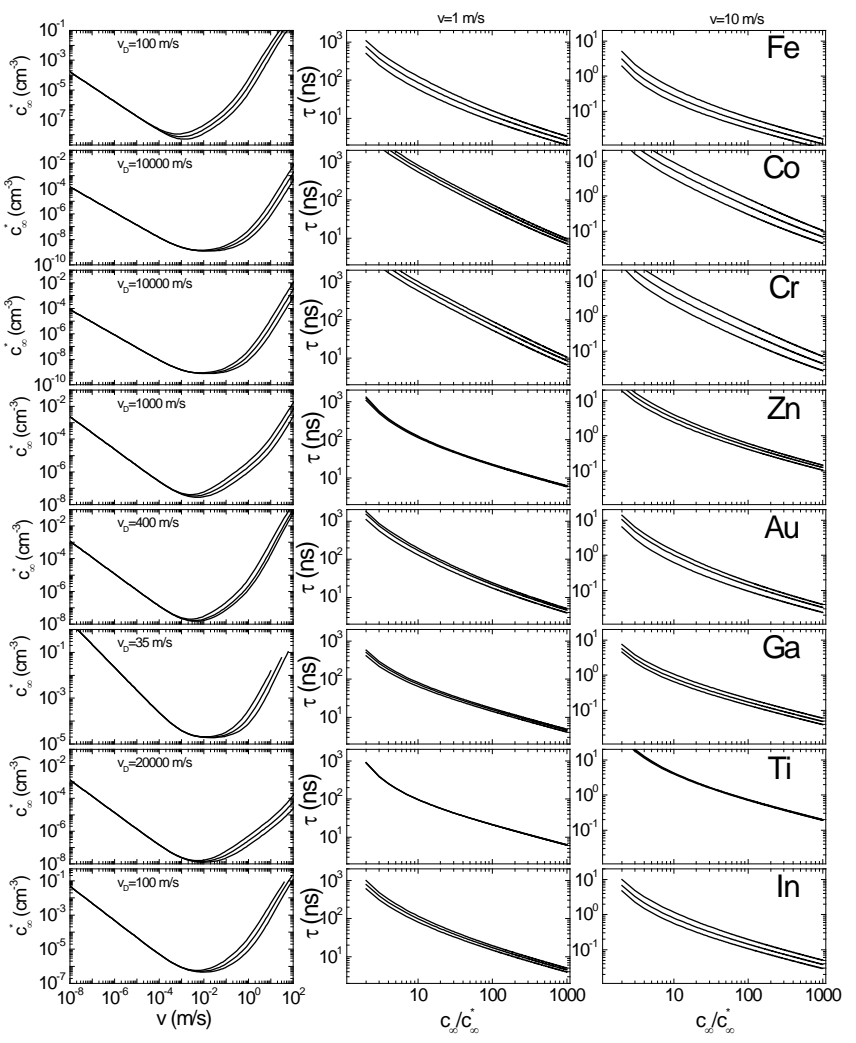

Fig. 2. Neutral stability curves (left column) for various impurities in Si (100). (exception: Ti was obtained for Si (111).) The amplification time, $\tau$, as a function of ratio $c_{\infty} / c_{\infty}^{*}$, at an interface speed of $1 \mathrm{~m} / \mathrm{s}$ (center column) and $10 \mathrm{~m} / \mathrm{s}$ (right column). All data were computed for three multiples of the best fit value for $v_{D}$, namely: $v_{D} / 2, v_{D}$, and $2 v_{D}$. In the left column, these three values resulted in the lower, middle, and upper curves, respectively. In the center and right columns, they produced the upper, middle, and lower curves, respectively. 


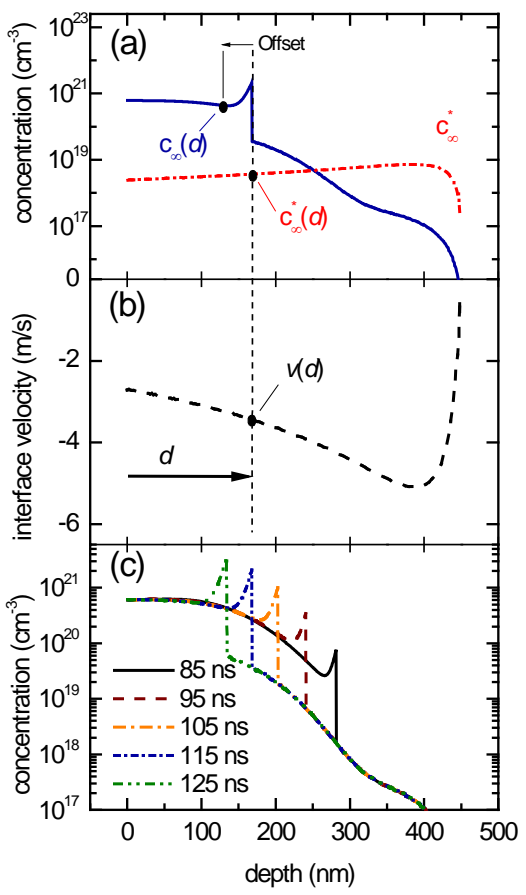

Fig. 3. (color online) Schematic illustration of the method for obtaining $\tau$ when the liquid-solid interface is at depth $d$. (a) and (b) The interface velocity $v(d)$ (dashed line) is obtained from 1D heat flow calculations, and the critical bulk liquid concentration (dash-dot line) is calculated using the interface speed as an input. The impurity concentration as a function of depth (solid blue line) is obtained from a $1 \mathrm{D}$ diffusion calculation, and $c_{\infty}$ is taken to be the impurity concentration in the liquid at a negative offset from the interface depth $d$, to avoid the tail that results from partitioning of the impurity at the interface. (c) Concentration depth profiles at various time steps under identical conditions to (a). For any time step, the bulk liquid concentration at depths shallower than the tail of the interface concentration maxima approximately falls onto the same curve; we use this curve as $c_{\infty}(d)$. 


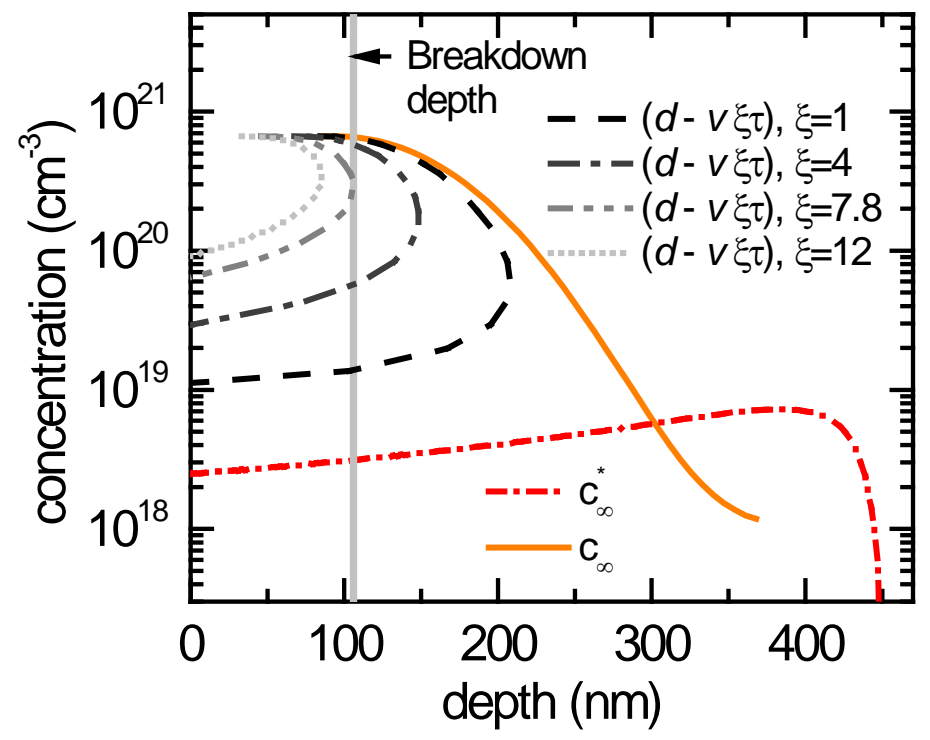

Fig. 4. (color online) Illustration of the method for obtaining the amplification coefficient $\xi$, for the case of In implanted at $200 \mathrm{keV}$ to a dose of $1 \times 10^{16} \mathrm{at} . / \mathrm{cm}^{2}$, and melted by a XeCl excimer laser at a fluence of $2 \mathrm{~J} / \mathrm{cm}^{2}$. The critical bulk liquid concentration (short dash-dot line) is calculated using the interface velocity $v$ vs. depth $d$ obtained from 1D heat flow calculations, and the bulk liquid concentration (solid line) is obtained from 1D diffusion calculations. The predicted breakdown depth is plotted for varying values of $\xi$. The ordinate value of these curves corresponds to the bulk liquid concentration when the interface is at depth $d$, and the abscissa, $d$ $-v \xi \tau$, is the predicted breakdown depth for a perturbation that begins at that depth $d$. Therefore, the rightmost value of the curve is the predicted breakdown depth for the layer. For In, the experimentally observed breakdown depth (vertical solid line) is $106 \mathrm{~nm}$. Setting $\xi=7.8$, the abscissa of the rightmost point on the breakdown depth curve is equal to the breakdown depth. 


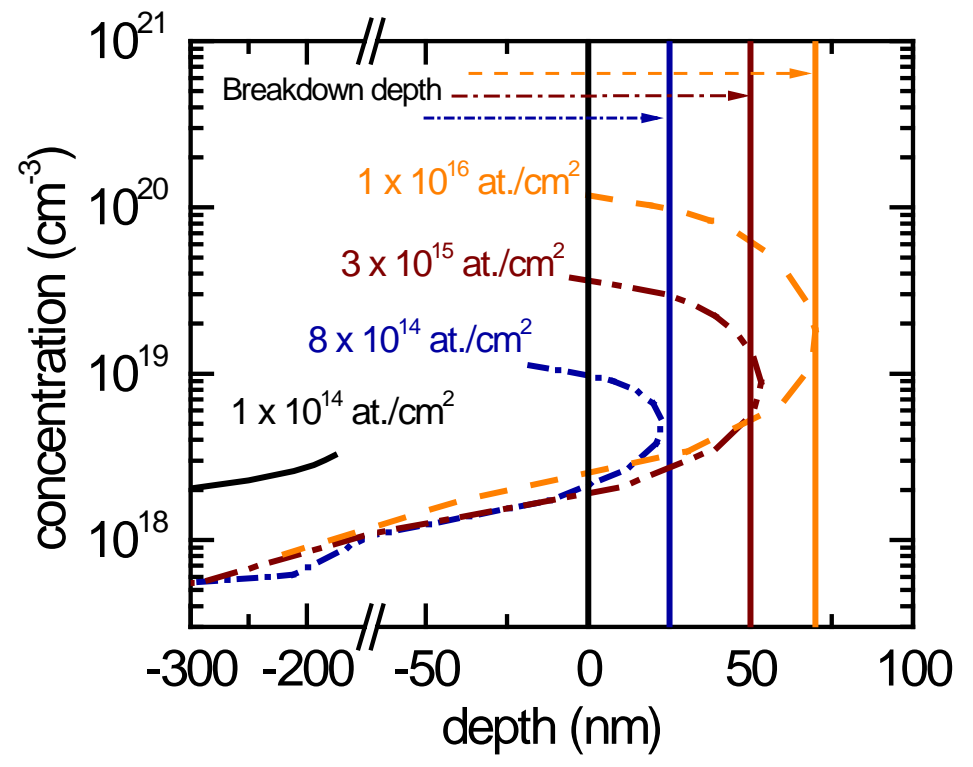

Fig. 5. (color online) Predicted breakdown depth $d-v \xi \tau$ for several implant doses of Ti in (111) $\mathrm{Si}$, for pulsed laser melting with an Nd:YAG laser at $0.75 \mathrm{~J} / \mathrm{cm}^{2}$. The same value of $\xi$ (4.06) is used for all doses. Vertical lines show the experimentally observed breakdown depths. The agreement between the rightmost point of each of the breakdown depth curves, and the corresponding experimental breakdown depth, is excellent. For the $1 \times 10^{14} \mathrm{at} . / \mathrm{cm}^{2}$ dose sample, the breakdown depth curve never reaches a positive depth, indicating that breakdown is not expected. This agrees with the experimental observation. 


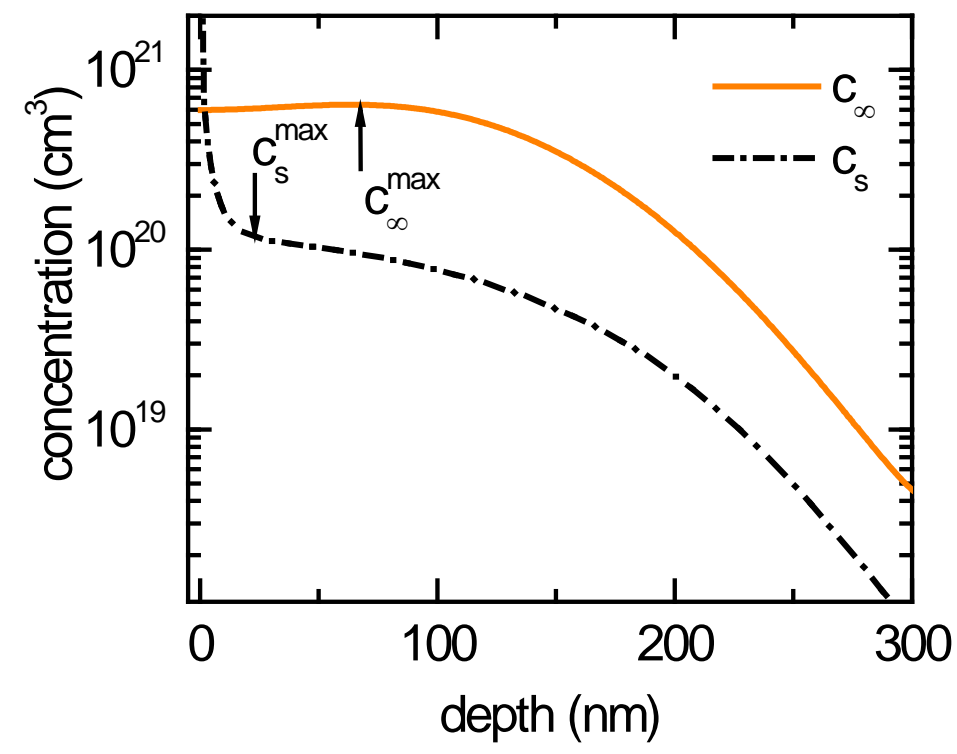

Fig. 6.(color online) Comparison of bulk liquid concentration at $45 \mathrm{~ns}$ (solid line) and solid concentration after solidification (dash-dot line) for In. The arrows denotes the peak of the bulk liquid concentration and solid concentration, which are used for calculation of the ratio $\zeta=\mathrm{c}_{\propto} / \mathrm{c}_{\mathrm{s}}$. 


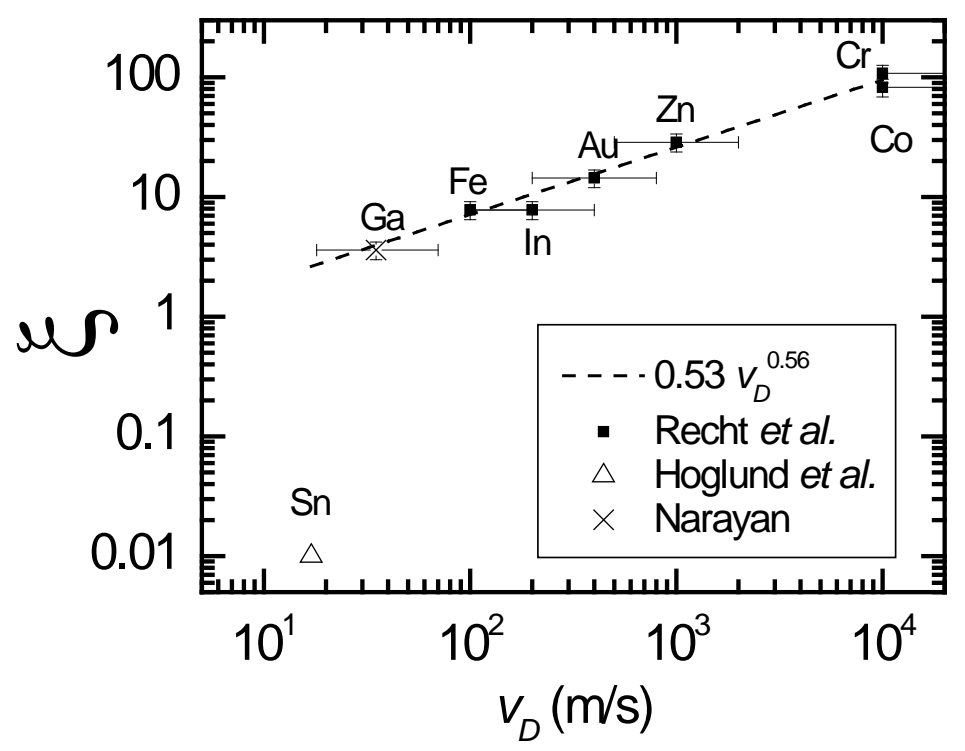

Fig. 7. Amplification coefficient $\xi$ as a function of $v_{D}$. With the exception of the Sn point from Ref. 19, the $\xi$ vs. $v_{D}$ data show power law behavior. The error bars show a possible factor of 2 error in $v_{D}$ and a possible error of $17 \%$ in $\xi$. (Note: fits to $v_{D}$ from Ref. 8 are lower bounds for Fe, Co, and Cr). 